\title{
PPM: A Privacy Prediction Model for Online Social Networks
}

CAILING DONG, University of Maryland, Baltimore County

HONGXIA JIN, Samsung Research America

BART P. KNIJNENBURG, Clemson University

Online Social Networks (OSNs) have come to play an increasingly important role in our social lives, and their inherent privacy problems have become a major concern for users. Can we assist consumers in their privacy decision-making practices, for example by predicting their preferences and giving them personalized advice? To this end, we introduce PPM: a Privacy Prediction Model, rooted in psychological principles, which can be used to give users personalized advice regarding their privacy decision-making practices. Using this model, we study psychological variables that are known to affect users' disclosure behavior: the trustworthiness of the requester/information audience, the sharing tendency of the receiver/information holder, the sensitivity of the requested/shared information, the appropriateness of the request/sharing activities, as well as several more traditional contextual factors.

CCS Concepts: •Security and privacy $\rightarrow$ Social network security and privacy; Privacy protections; $\bullet$ Human-centered computing $\rightarrow$ Social network analysis;

Additional Key Words and Phrases: Privacy prediction; decision making; classification; machine learning; Online Social Networks (OSNs)

\section{INTRODUCTION}

The rising popularity of Online Social Networks (OSNs) has ushered in a new era of social interaction that increasingly takes place online. Pew Research reports that $72 \%$ of online American adults maintain a social network profile [Brenner and Smith 2013], which provides them with a convenient way to communicate online with family, friends, and even total strangers. To facilitate this process, people often share personal details about themselves (e.g. likes, friendships, education and work history). Many users even share their current activity and/or real-time location. However, all this public sharing of personal and sometimes private information may increase security risks (e.g., phishing, stalking [Gross and Acquisti 2005; Al Hasib 2009]), or lead to threats to one's personal reputation [Baker et al. 2011]. It is therefore no surprise that privacy aspects of OSN use has raised considerable attention from researchers, OSN managers, as well as users themselves.

The privacy dilemma OSN users face is the choice between sharing their information (which may result in social benefits [Ellison et al. 2007; Joinson 2008; DiMicco et al. 2008; Lampe et al. 2014]) and keeping it private or restricted to certain users only (thereby protecting their privacy). To help users with this decision, experts recommend giving users comprehensive control over what data they wish to share, and providing them with more transparency regarding the implications of their decisions [Acquisti and Gross 2006, Benisch et al. 2011; Tang et al. 2012; Tang et al. 2010, Toch et al. 2010]. Advocates of transparency and control argue that it empowers users to regulate their privacy at the desired level: without some minimum level of transparency and control, users cannot influence the risk/benefit tradeoff. Moreover, people can only make an informed tradeoff between benefits and risks if they are given adequate information [Lederer et al. 2004, Sadeh et al. 2009].

The privacy decisions on ONSs are so numerous and complex, that users often fail to manage their privacy effectively. Many users avoid the hassle of using the "labyrinthian" privacy controls that Facebook provides [Compañó and Lusoli 2010, Reports 2012], and those who make the effort to change their settings do not even seem to grasp the implications of their own privacy settings [Liu et al. 2011; Madejski et al. 2012]. There is strong evidence that transparency and control do not work well in practice, and several prominent privacy scholars have denounced their effectiveness in helping users to make better privacy decisions [Barocas and Nissenbaum 2009, Nissenbaum 2011, Solove 2013].

Are there more effective ways to assist consumers in their privacy decision-making practices? A solution that has recently been proposed, is to learn users' privacy preferences and subsequently 
give them user-tailored decision support [Knijnenburg 2013]. To this end, in this paper we introduce PPM: a comprehensive privacy prediction model that can be applied in a multitude of privacy decision making scenarios. The model is theoretically grounded in psychological research that has investigated the key variables known to affect users' disclosure behavior. Consequently, PPM can be applied to a wide range of OSNs, and arguably even other privacy-sensitive systems such as e-commerce systems.

A practical application of PPM's predictions would be to provide automatic initial default settings in line with users' privacy preferences (e.g., by default, it discloses Mary's location to her best friends on weekends, but it does not disclose John's location to his boss when he is on vacation). These "smart defaults" could alleviate the burden of making numerous complex privacy decisions, while at the same time respecting users' inherent privacy preferences. Balebako et al. [Balebako et al. 2011] argue that the help provided via such machine learning systems can be seen as "adaptive nudges". Indeed, Smith et al. [Smith et al. 2013] argue that "Smart defaults can become even smarter by adapting to information provided by the consumer as part of the decisionmaking process." (p. 167)

In this paper we will formally define PPM and then use it to comprehensively study the important psychological and contextual factors that affect privacy decision making on OSNs, with the ultimate goal of assisting users to make appropriate privacy decisions. Specifically, we validate PPM in multiple scenarios, testing the effect of several psychological antecedents of information disclosure behavior - the trustworthiness of the requester/audience, the sharing tendency of the user, the sensitivity of the information, the appropriateness of the request/disclosure-as well as several more traditional contextual factors on data collected on Twitter, Google+, and a location sharing preference study. We provide a comparative evaluation of the importance of each of these factors in determining users' privacy decisions.

\section{RELATED WORK}

\subsection{Privacy Decision Making}

A majority of OSN users takes a pragmatic stance on information disclosure [Sheehan 2002, Taylor 2003, Consolvo et al. 2005]. These "pragmatists" [Harris et al. 1998] have balanced privacy attitudes: they ask what benefits they get, and balance these benefits against risks to their privacy interests [Consolvo et al. 2005]. This decision process of trading off the anticipated benefits with the risks of disclosure has been dubbed privacy calculus [Culnan 1993, Laufer and Wolfe 1977]). In making this tradeoff, these users typically decide to share a subset of their personal information with a subset of their contacts [Olson et al. 2005, Consolvo et al. 2005, Lewis et al. 2008, Lampe et al. 2008.

The term privacy calculus makes it sound like users make "calculated" decisions to share or withhold their personal information. In reality though, these decisions are numerous and complex, and often involve uncertain or unknown outcomes [Knijnenburg and Kobsa 2013]. Acquisti and Grossklags [Acquisti and Grossklags 2005] identified incomplete information, bounded rationality, and systematic psychological deviations from rationality as three main challenges in privacy decision making. Consequently, people's privacy behavior is far from calculated or rational. Most OSN users share much more freely than expected based on their attitudes [Acquisti and Gross 2006, Becker and Pousttchi 2012; Knijnenburg et al. 2013b; Acquisti and Grossklags 2005] (a disparity that has been labeled the "privacy paradox" [Norberg et al. 2007]). In [Liu et al. 2011], the authors quantified this disparity between the desired and actual privacy settings, and found that users' privacy settings match users' expectations only $37 \%$ of the time and oftentimes people shared more than they expected.

\subsection{Predicting Privacy Decisions}

When left to their own devices, users thus seem particularly inept at making even the simplest privacy decisions in a rational manner [Knijnenburg et al. 2013b], and many users actively try to 
avoid the hassle of making such decisions [Compañó and Lusoli 2010]. Knijnenburg et al. have recently proposed a way to circumvent users' unwillingness or inability to make accurate privacy decisions: if one can predict users' privacy preferences, one can give them user-tailored decision support [Knijnenburg 2013] in the form of recommendations [Knijnenburg and Jin 2013] or adaptive defaults [Knijnenburg and Kobsa 2014], which would help to limit the number and complexity of privacy decisions that OSN users have to make. Regarding the first step of this proposal (i.e., predicting users' privacy decisions), scientists have had modest success using various machine learning practices.

For example, Ravichandran et al. Ravichandran et al. 2009] applied k-means clustering to users' contextualized location sharing decisions to come up with a number of default policies. They showed that a small number of default policies learned from users' contextual location sharing decisions could accurately capture a large part of their location sharing preferences. Similarly, Sadeh et al. [Sadeh et al. 2009] applied a k-nearest neighbor (kNN) algorithm and a random forest algorithm to accurately predict users' privacy preferences in a location-sharing system based on the type of recipient and the time and location of the request. They showed that users had difficulties setting their privacy preferences, and that the applied machine learning techniques could help users in specifying more accurate disclosure preferences. Pallapa et al. [Pallapa et al. 2014] proposed a system that determines the level of privacy required in new situations based on the history of interaction between users. They demonstrated that this solution can deal with the rise of privacy concerns while at the same time efficiently supporting users in a pervasive system full of dynamic and rich interactions. Finally, in a social network context, Fang and LeFevre [Fang and LeFevre 2010] developed a privacy wizard that is able to configure users' privacy settings automatically and accurately with a machine learning model that they developed. The wizard removes the burden of setting privacy settings using tools that most users would otherwise find too difficult to understand and use.

\subsection{Psychological Antecedents of Privacy Decisions}

While machine learning studies have had modest success predicting users' privacy decisions, their results have been scattered; each work only considers a small subset of (one or two) contextual factors, in the context of a single OSN. In this paper, we therefore make an effort to integrate new and existing privacy prediction factors into a single comprehensive model. To make this model generalizable across a wide range of OSNs (and, arguably, information systems in general), we theoretically ground this framework in psychological research that has identified factors that consistently influence the outcomes of users' privacy decisions.

Existing work has found several psychological factors that influence users' decision making process. For example, Adams identified three major factors that are key to users' privacy perceptions: information sensitivity, recipient and usage [Adams 2000. These factors are in line with Nissenbaum's theory of contextual integrity [Nissenbaum 2009], which argues that disclosure depends on context, actors, attributes, and transmission principles (cf. usage and flow constraints). Based on these theories and other existing research, we identify the following factors:

- The user: sharing tendency. The most widely accepted finding in privacy research is that users differ in their innate tendency to share personal information [Sheehan 2002, Taylor 2003. Consolvo et al. 2005]. Most prominently, Westin and Harris [Harris et al. 1998] developed a privacy segmentation model which classifies people into three categories: privacy fundamentalists, pragmatists, and unconcerned. Recent work has demonstrated that this categorization might be overly simplistic [Woodruff et al.2014]. In this light, Knijnenburg et al. [Knijnenburg et al. 2013a] demonstrated that people's disclosure behavior is in fact multidimensional, that is, different people have different tendencies to disclose different types of information (see also [Olson et al. 2005).

- The information: sensitivity. Several studies have found that different types of information have different levels of sensitivity, and that users are less likely to disclose more sensitive information. For instance, Consolvo et al. [Consolvo et al. 2005] and Lederer et al. [Lederer et al. 2003] both 
found that users are more willing to share vague information about themselves than specific information. Similarly, some privacy decision-making studies found that their manipulation only works for sensitive information [Acquisti et al. 2012]. Note that users occasionally differ in what information they find most sensitive; i.e., Knijnenburg et al. [Knijnenburg et al. 2013a] find that some users are less willing to publicly share their location than their Facebook posts, while this preference is reversed for others. Generally speaking, though, broad universally applicable levels of information sensitivity can be discerned (e.g. credit card information is much more sensitive than age and gender), and users are typically less willing to share the more sensitive information.

- The recipient: trustworthiness. Many studies highlight the trustworthiness of the recipient of the information as an important factor [Consolvo et al. 2005, Hsu 2006, Johnson et al. 2012, Norberg et al. 2007; Olson et al. 2005, Toch et al. 2010]. Lederer et al. [Lederer et al. 2003] even found that this factor overshadows more traditional contextual factors in terms of determining sharing tendency. Indeed, OSN users tend to restrict access to their profiles by sharing certain information with certain people only [Kairam et al. 2012, Madden 2012], and OSNs have started to accommodate this factor by introducing the facility to categorize recipients into "groups" or "circles". Research shows that users make extensive use of this facility, albeit often ineffectively [Kairam et al. 2012, Knijnenburg and Kobsa 2014: Watson et al. 2012].

- The context: appropriateness. Nissenbaum's theory of contextual integrity [Nissenbaum 2009] posits that context-relevant norms play a significant role in users' sharing decisions. Specifically, the theory suggests that disclosure depends on whether it is deemed appropriate or inappropriate in that specific context. Indeed, several scholars argue that the appropriateness of the information request/disclosure plays an important role in determining users' sharing decisions [Nissenbaum 2009, Bansal et al. 2008, Borcea-Pfitzmann et al. 2011, Xu et al. 2008]. Evaluations of appropriateness are based on users' perception of whether there is a straightforward reason why this recipient should have access to this piece of information in this specific context. This reason is often related to a stated or imagined usage scenario (e.g. I share my work e-mail address [information] with my colleague Dave [recipient] so that he can send me work-related documents [usage]).

\section{THE PRIVACY PREDICTION MODEL}

The aforementioned psychological antecedents of privacy decisions highlight the incredible complexity of the privacy calculus. For each privacy decision, users need to estimate the benefits and risks of disclosure by determining and then integrating all these components: their sharing tendency, the sensitivity of the information, the trustworthiness of the recipient, and the appropriateness of the disclosure in context. Arguably, this is a mentally challenging activity, and it is no surprise that our boundedly rational minds are unable to cope with such complex decisions [Acquisti and Grossklags 2005].

However, the cited work suggests that these antecedents do hold considerable predictive value, meaning that while the privacy calculus may be mentally unattainable, it may very well be computationally feasible to make consistent predictions of users' preferred privacy decisions based on these antecedents. Indeed, machine learning algorithms are particularly suitable to provide such consistent predictions based on a multitude of anteceding factors. As mentioned, such algorithms have been used in limited cases to predict privacy behaviors [Ravichandran et al. 2009, Sadeh et al. 2009, Pallapa et al. 2014, Fang and LeFevre 2010]. In this section, we will formalize and operationalize this approach. Specifically, we will first provide a generic formal definition of the PPM, and discuss a range of algorithms that can be used to implement it. Subsequently, we will operationalize the PPM by proposing an expandable set of behavioral analogs of the psychological antecedents, which allow us to unobtrusively measure these antecedents.

\subsection{Theory: A Comprehensive Model Based on Psychological Antecedents}

Formally, PPM models the probability of disclosure $p(D)$ by user $u$ of item $i$ to recipient $r$ in context $c$ as a function of the user's disclosure/sharing tendency $\bar{D}_{u}$, the sensitivity of the item $S_{i}$, 
the trustworthiness of the recipient $T_{r}$, and the appropriateness of the disclosure in this specific context $A_{c}$ :

$$
p\left(D_{u i r c}\right)=f\left(\overline{D_{u}}, S_{i}, T_{r}, A_{c}\right)
$$

Overly simplistic implementations of this model are the loglinear additive model (Eq. 2) and the full factorial loglinear model (Eq. 3):

$$
\begin{gathered}
\ln \left(\frac{p\left(D_{\text {uirc }}\right)}{1-p\left(D_{u i r c}\right)}\right)=\alpha+\beta_{1} \overline{D_{u}}+\beta_{2} S_{i}+\beta_{3} T_{r}+\beta_{4} A_{c} \\
\ln \left(\frac{p\left(D_{u i r c}\right)}{1-p\left(D_{u i r c}\right)}\right)= \\
+\beta_{7} \overline{D_{u}} A_{c}+\beta_{8} S_{i} T_{r}+\beta_{9} S_{i} A_{c}+\beta_{10} T_{r} A_{c}+\beta_{11} \overline{D_{u}} S_{i} T_{r} \\
+\beta_{12} \overline{D_{u}} S_{i} A_{c}+\beta_{13} \overline{D_{u}} T_{r} A_{c}+\beta_{14} S_{i} T_{r} A_{c}+\beta_{15} \overline{D_{u}} S_{i} T_{r} A_{c}
\end{gathered}
$$

Machine learning algorithms can provide more sophisticated implementations, such as decision trees, bayesian models, and support vector machines. We will try several of these implementations in our results section.

\subsection{Practice: Large-scale Prediction Using Behavioral Analogs}

An essential step towards operationalizing PPM is to measure or estimate the parameters $\overline{D_{u}}, S_{i}$, $T_{r}$, and $A_{c}$. This is not a trivial task: these psychological antecedents are hard to quantify, especially on the large scale needed for successful machine learning. Current work defines three ways in which a system can do this:

- Ask the user. The simplest solution is to directly ask the user, either by having them specify exact values for each parameter, or by allowing them to make a broad classification (e.g. when the system asks the user to categorize a recipient as a "friend" or "acquaintance", cf. [Kairam et al. 2012, Knijnenburg and Kobsa 2014, Watson et al. 2012]). This approach is arguably the most accurate (no estimation is involved), but it simply shifts the user's burden from the disclosure decision to the parameter specification (cf. Knijnenburg and Jin [Knijnenburg and Jin 2013] show that this approach does not improve user satisfaction).

- Derive the values. Another option is to derive these values from static user variables, such as when the system uses proxy variables, such as when it uses user characteristics (e.g., age, gender, cultural background, or mobile Internet usage) or attitudes (e.g., privacy concerns) to determine users' disclosure profile. This approach is unobtrusive, but rather inaccurate, since these proxy variables are often not context-specific (cf. Knijnenburg et al. [Knijnenburg et al. 2013a] show that such variables are not very capable of distinguishing between different disclosure profiles).

- Estimate with available data. Finally, a system can forego the parameters, and simply try to estimate users' disclosure behaviors based on any available contextual variables. As a finergrained and thus ultimately more precise method, most existing work uses this approach (cf. [Ravichandran et al. 2009, Sadeh et al. 2009, Pallapa et al. 2014, Fang and LeFevre 2010]). Note, though, that not every available contextual variable has equal predictive value, and using too many contextual variables can lead to overfitting. As such, this approach lacks clear guidance on how to select the most appropriate contextual variables for the estimation process.

The PPM approach described in this paper takes a spin on the "estimate with available data" method by specifying specific types of data as behavioral analogs of the established psychological antecedents. In the following sections, we identify these behavioral analogs in several datasets. Consequently we integrate all factors into a privacy decision making prediction model that can help OSN users to make better privacy decisions. 


\section{DATA COLLECTION}

Friend requests are the most common and direct way to get access to a user's information in many OSNs. Accepting a friend request discloses at least a part of one's profile and online activities to the requester, so the acceptance or rejection of a friend request is an important privacy decision. In our study, we collected two real-life datasets from Twitter and Goolge+, targeting on the "Friend requests" activities to simulate the information disclosure behavior. Besides, location-sharing has gained popularity both in stand-alone apps (e.g. Foursquare, Glympse) and as a feature of existing OSNs (e.g. location-tagging on Facebook and Twitter), which is an information disclosure activity that is particularly strongly influenced by privacy concerns [Page et al. 2012, Zickuhr 2012]. Unfortunately, existing location-sharing datasets often do not extend beyond check-in behaviors. We thus created three location-sharing datasets based on a study with manually-collected rich location sharing preferences that includes twenty location semantics, three groups of audiences and several contextual factors such as companion and emotion [Dong et al. 2015]. In the following, we will describe how we collect each dataset and identify the above-mentioned behavioral analogs in these datasets.

\subsection{Twitter Dataset}

Our Twitter dataset consists of a set of Twitter users crawled and classified as legitimate users by Lee et al. [Kyumin Lee and Caverlee 2011]. We measured the behavioral analogs of our psychological antecedents as follows:

4.1.1. Disclosure behavior. In our datasets from both Twitter and Google+, we study users' responses to "Friend requests" as our target information disclosure behavior. Two general friendship mechanism exist on social networks: in bilateral friendship requests (e.g. Facebook) friendships are reflexive, and a friendship is only established after the user accepts the request. While this is the "cleanest" version of our scenario, the requests themselves are not accessible through the Facebook API, effectively making it impossible to observe rejected requests. Twitter and Google+, on the other hand, use unilateral friendship requests: users do not need permission to "follow" or "add to circle" other users, making one-sided "friendships" possible.

Users who are followed/added to a circle may respond in one of three ways: (1) they may reciprocate the request by following/adding the requester back; (2) they may delete or block the requester; (3) or they may do nothing and simply leave the friendship one-sided. Behavior 1 is observable as a separate, subsequent friendship request; behaviors 2 and 3 are indistinguishable using the Twitter and Google+ APIs. However, since users are notified of being followed, we argue that users will most commonly follow/add the requester back if they accept the request, and otherwise simply delete or block the requester. In other words: behavior 3 occurs rarely for users who are not celebrities (who typically have much more followers than followees). Therefore, our work is based on the assumption that when a user follows the requester back the request is accepted, otherwise it is rejected.

To measure this behavior, we extracted each user $u$ 's profile item settings, and following and follower lists. Each friendship request is represented by a tuple $\langle f(u), f(v), f(u, v), l\rangle$, where $u$ is the requester, $v$ is the receiver and $l$ is the decision label indicating if $v$ accepts (1) or rejects (0) $u$ 's request. $f(u)$ and $f(v)$ are collections of features associated with $u$ and $v$ respectively, and $f(u, v)$ represents the relationship between $u$ and $v$. We classify friend request decisions as follows:

Definition 4.1 (Disclosure behavior). For each friend $u_{f}$ on $u$ 's following list, if $u_{f}$ is also in $u$ 's followers list, that is, if $u_{f}$ also follows $u$, we say that $u_{f}$ accepted $u$ 's request, otherwise $u_{f}$ rejected $u$ 's request.

Consequently, we extracted $u_{f}$ 's profile items and following and follower lists as well. This results in a total of 17,118 users in our dataset. We removed "verified" users, since most of such users are celebrities who often have many more followers than followees; our definition of "accepting/rejecting a friend request" will arguably not hold for such users. 
Note that based on our Twitter dataset we are unable to distinguish who follows whom first; when two users $u_{1}$ and $u_{2}$ follow each other, this results in two records $<f\left(u_{1}\right), f\left(u_{2}\right), f\left(u_{1}, u_{2}\right), 1>$ and $<f\left(u_{2}\right), f\left(u_{1}\right), f\left(u_{2}, u_{1}\right), 1>$. Only one of these describes the actual reciprocation of a friend request, the other is spurious. In the Twitter dataset this results in a noisy dataset where only half of the signals are real. In the Google+ dataset we present below, we are able to untangle the chronological order of friend requests. This dataset thus arguably provides more accurate results.

4.1.2. Sharing tendency of the information holder. FollowTendency is a behavioral analog of users' disclosure/sharing tendency, defined as the relative number of people they follow. Formally speaking:

Definition 4.2 (Sharing tendency). followTendency $=\frac{\# \text { following }}{\# \text { follower }+\# \text { following }}$

4.1.3. Trustworthiness of the recipient. Our behavioral analog of the recipient's trustworthiness is based on the intuition that a user with relatively many followers is likely to have a higher reputation, and thus more trustworthy. Formally speaking:

$$
\text { Definition } 4.3 \text { (Trustworthiness). trustworthiness }=\frac{\# \text { follower }}{\# \text { follower }+\# \text { following }}
$$

4.1.4. Sensitivity of the requested information. We argue that the sensitivity $S_{i}$ of a Twitter user $u$ 's profile item $i$ depends on how common the user's value of $i$ is in the population: the more common the value, the less sensitive the information. Formally speaking:

Definition 4.4 (Sensitivity). Suppose a profile item $i$ has $m$ possible settings $\left\{i_{1}, i_{2}, \ldots, i_{m}\right\}$ $(m \geq 1)$. The distribution of different settings over the whole population is $P^{i}=$ $\left\{p_{i_{1}}, p_{i_{2}}, \ldots, p_{i_{m}}\right\}$, where $0 \leq p_{i_{j}} \leq 1$ and $\sum_{j=1}^{m} p_{i_{j}}=1$. If user $u$ sets his $/$ her profile item $i$ as $i_{k}(1 \leq k \leq m)$, the sensitivity value of $S_{i}=\frac{1}{p_{i_{k}}}$.

On Twitter, users have the option to set profile items URL, GEO and Protected. GEO and Protected are boolean values indicating whether the user has enabled the automatic geo-tagging of her tweets, and whether her profile is protected (i.e. her updates can only be followed with explicit consent from the user). URL is a field that users can use to enter a personal website. We categorize its value as either blank, a personal URL (linking to Facebook or LinkedIn), or an other URL. We calculate the corresponding sensitivity scores for these items and use them as our behavioral antecendents of sensitivity.

4.1.5. Appropriateness of the request. Friendship requests are more appropriate if there is a lot of existing overlap between the two users' networks. Formally speaking:

Definition 4.5 (Appropriateness). The approrpiateness of a friend request of user $v$ to user $u$ depends on the overlap between their networks, which can be measured with the following indicators:

$$
\begin{aligned}
& \text { - JaccardFollowing }(u, v)=\frac{\mid \text { followings }(u) \cap \text { followings }(v) \mid}{\mid \text { followings }(u) \cup \text { followings }(v) \mid} \\
& \text { - JaccardFollower }(u, v)=\frac{\mid \text { followers }(u) \cap \text { followers }(v) \mid}{\mid \text { followers }(u) \cup \text { followers }(v) \mid} \\
& \text { - comFollowing }(u)=\frac{\# \text { commonFollowing }}{\# \text { following }(u)} \\
& \text { - comFollower }(u)=\frac{\# \text { commonFollower }}{\# \text { follower }(u)} \\
& \text { - comFollowing }(v)=\frac{\# \text { commonFollowing }}{\# \text { following }(v)} \\
& \text { - comFollower }(v)=\frac{\# \text { commonFollower }}{\# \text { follower }(v)}
\end{aligned}
$$

\subsection{Google+ Dataset}

Gong et al. [Gong et al. 2011] crawled the whole evolution process of Google+, from its initial launch to public release. The dataset consists of 79 network snapshots, these stages can be used to uncover a rough chronological account of friendship creation (i.e. users adding each other to their 
circles). We focus on the first two stages to build our dataset, where "who sends the friend request to whom first" can be identified by the stage ids. In total, the set contains 3,481,544 active user: in stage 0 and 14,289,211 in stage 1 .

We used the same factors in our Twitter dataset to measure the behavioral analogs of our psychological antecedents. Specifically, we use the "add to circle" activity to simulate the information disclosure behavior. Each instance in our Google+ dataset is a tuple $\langle f(u), f(v), f(u, v), l>$, where $l$ indicates $v$ ' decision to reciprocate $u$ 's request (1) or not (0).

We use the same behavioral analogs as those defined in Twitter dataset for sharing tendency, trustworthiness, sensitivity, and the appropriateness of the request. For sensitivity, we use profile attributes available on Google+, namely Employer, Major, School and Places that are either publicly displayed (1) or not (0). We thus calculate the following four behavioral analogs for sensitivity: (1) $S$ (Employer), (2) S(Major), (3) S(School) and (4) S(Places).

\subsection{Location Sharing Datasets}

Our location sharing datasets are built based on a survey on location sharing preferences. We conducted this survey by recruiting 1,088 participants using Amazon Mechanical Turk2. We restricted participation to US Turk workers with a high worker reputation who had previously used a form of location sharing services. The demographics are shown in Table $\square$

\begin{tabular}{|c|c|}
\hline Attribute & Distribution \\
\hline Age & $\begin{array}{c}18 \text { to } 24(21.42 \%), 25 \text { to } 34(45.23 \%), \\
35 \text { to } 44(20.23 \%), 45 \text { to } 54(5.95 \%) \\
55 \text { to } 64(7.14 \%)\end{array}$ \\
\hline Gender & male $(57.14 \%)$, female $(42.85 \%)$ \\
\hline Marriage & married $(40.47 \%)$, not married $(59.52 \%)$ \\
\hline
\end{tabular}

Table I: Demographics of the participants in the location sharing preference study.

We specifically asked the participants about their privacy concern in the location sharing survey. The distribution of their claimed privacyLevel is: Very Concerned (39\%), Moderately (41\%), Slightly (15\%), Not Care (5\%). Consistent with previous research, as many as $80 \%$ of the participants claimed to be moderately or very concerned about their privacy Harris et al. 1998, Ho et al. 2009.

4.3.1. Disclosure behavior. We constructed our dataset by requesting users' feedback to systematically manipulated location sharing scenarios. In each scenario, participants were asked to indicate whether they would share their location with three different types of audience: Family, Friend and Colleague.

We ran 5 different studies to collect our data:

- In study 1 , each scenario consisted of one of twenty location semantics supported by Google Places3: Airport, Art Gallery, Bank, Bar, Bus Station, Casino, Cemetery, Church, Company Building, Convention Center, Hospital, Hotel, Law Firm, Library, Movie Theater, Police Station, Restaurant, Shopping Mall, Spa and Workplace.

- In study 2, each scenario consisted of a location, plus a certain time: on a weekday during the day, on a weekday at night, on the weekend.

- In study 3, each scenario consisted of a location, plus a companion: alone, with family, with friends, or with colleagues.

\footnotetext{
${ }^{1}$ We call a user with at least one following as an "active user".

${ }^{2}$ https://www.mturk.com/mturk/

${ }^{3} \mathrm{https}: / /$ developers.google.com/places/
} 
- In study 4, we combined location, time and companion in each scenario.

- In study 5, each scenario consisted of a location, plus an emotion: positive or negative.

Some of these contextual variables match features available on location sharing services, such as "who are you with", and emotion icons on Facebook. In total, five different scenarios were presented to the user, as shown in Table II. Each participant was randomly assigned ten scenarios with different combinations of location and contextual information.

\begin{tabular}{|c|c|c|c|}
\hline Study & Scenario & \#Participants & \#Records \\
\hline \hline 1 & location & 84 & 840 \\
2 & location+time & 133 & 1376 \\
3 & location+ companion & 244 & 2440 \\
4 & location + time + companion & 510 & 4969 \\
5 & location + emotion & 117 & 1170 \\
\hline
\end{tabular}

Table II: Experimental settings of location sharing preference study.

For each targeted group of audience $V$ (Family, Friend and Colleague), we collect the associated sharing records represented by tuples $<f(u), f(u, V, l o c), f(l o c), l(V)>$, which results in 3 location sharing datasets. $f(u)$ represents the user's features. $f(u, V, l o c)$ describes the relationship between the three parties, i.e., user, audience and location. As the sharing information is the given location, we specifically include the features $f(l o c)$ with regard to the current location loc into each tuple. $l(V)$ is the decision label indicating if $u$ shares her location with audience $V(1)$ or not $(0)$.

4.3.2. Sharing tendency of the information holder. In this "voluntarily information sharing" scenario, sharing tendency of the information holder largely influences his/her privacy decisions. In our location sharing preference study, each user $u$ only has one sharing option to each group of audiences under a specific scenario. That is, we do not have the "historical" sharing records of $u$ with the same scenario to predict the current or future privacy decision. Therefore, we choose to use other users' sharing behavior to estimate individual $u$ 's sharing probability.

One type of estimation on sharing tendency is based on a specific feature $Q$ over all the populations in the dataset, i.e., overall sharing probability, represented by $p^{u}(Q)$. That is, $p^{u}(Q)$ represents the sharing tendency of $u$ based on feature $Q$. We estimate it by the sharing probability of the users in the given dataset $\mathrm{R}$ who have the same feature value of $Q$ with $u$, regardless of other information such as sharing audience, current location, etc. Formally speaking,

Definition 4.6 (overall sharing probability). Suppose a feature $Q$ has $m$ possible values $\left\{q_{1}, q_{2}, \ldots, q_{m}\right\}(m \geq 1)$. The sharing probability of the users with different feature values on $Q$ over the whole records $R$ is $P^{u}(Q)=\left\{p_{q_{1}}, p_{q_{2}}, \ldots, p_{q_{m}}\right\}$, where $0 \leq p_{q_{i}} \leq 1$ and $\sum_{i=1}^{m} p_{q_{i}}=1$. That is, $p_{q_{i}}$ represents the sharing probability of the users with $q_{i}$ as the value of feature $Q$. If user $u$ 's feature value on $Q$ is $q_{k}(1 \leq k \leq m)$, the overall sharing probability of $u$ based on feature $Q$ is $p^{u}(Q)=p_{q_{k}}$.

In this study, we consider the disclosure tendency of $u$ from the four aspects: demographic features age, gender and marriage, as well as the claimed privacyLevel. It results in four types of overall sharing probability, i.e., $p^{u}$ (age), $p^{u}$ (gender), $p^{u}$ (marriage) and $p^{u}$ (privacyLevel).

Besides the specific feature $Q$, the other type of estimation on sharing tendency also considers the contextual variable $\alpha$. We call such estimation as $\alpha$-conditional sharing probability and denoted it as $p_{\alpha}^{u}(Q)$. We use the sharing probability of the users in $\mathrm{R}$ with the same attribute value on $Q$ who have been under the same scenario $\alpha$ to estimate $u$ 's sharing probability. Formally speaking,

Definition 4.7 ( $\alpha$-conditional sharing probability). Suppose an attribute $Q$ has $m$ possible values $\left\{q_{1}, q_{2}, \ldots, q_{m}\right\}(m \geq 1)$. The sharing probability of the users with different attribute values on $Q$ over the whole set of sharing records $R^{\alpha}$ under scenario $\alpha$ is $P_{\alpha}^{u}(Q)=\left\{p_{q_{1}}^{\alpha}, p_{q_{2}}^{\alpha}, \ldots, p_{q_{m}}^{\alpha}\right\}$, 
where $0 \leq p_{q_{i}}^{\alpha} \leq 1$ and $\sum_{i=1}^{m} p_{q_{i}}^{\alpha}=1$. If user $u$ 's feature value on scenario $Q$ is $q_{k}(1 \leq k \leq m)$, the $\alpha$-conditional sharing probability of $u$ based on $Q$ under $\alpha$ is $p_{\alpha}^{u}(Q)=p_{q_{k}}^{\alpha}$.

In this study, we set $\alpha$ as the current location loc or the audience $V$, and consider companion, emotion, time as well as the $l o c$ and $V$ as possible contextual variables. That is, we will consider the following $\alpha$-conditional sharing probabilities: $p_{l o c}^{u}($ companion $), p_{l o c}^{u}($ emotion $), p_{l o c}^{u}($ time $)$, $p_{V}^{u}$ (companion), $p_{V}^{u}$ (emotion), $p_{V}^{u}($ time $), p_{l o c}^{u}(V)$ and $p_{V}^{u}(l o c)$.

When building the PPM, we choose the corresponding overall sharing probability and $\alpha$ conditional sharing probability to represent the disclosure/sharing tendency $\overline{D_{u}}$ according to the different scenarios listed in Table

4.3.3. Trustworthiness of the recipient. As location sharing is a voluntarily sharing activity, the information holder usually makes the privacy decisions partially based on the trustworthiness of the audience. Typically, the higher of the probability $u$ is willing to share to the given type of audience $V$, the higher trustworthiness of $V$ is believed by $u$. According to the above definitions on sharing tendency, we can formally define trustworthiness as follows:

Definition 4.8 (Trustworthiness). The trustworthiness of $u$ to the sharing audience $V$ is estimated by the audience-conditional sharing probability of $u$ under the contextual variable $V$ without consider other features. That is, trustworthiness $(V)=p_{V}^{u}(\cdot)$.

4.3.4. Sensitivity of the shared location. The sensitivity of the location being shared is of vital importance to the privacy decisions. Usually, the more people is willing to share a location $l o c$, the less they think the location is sensitive. Thus, we can use the location-conditional sharing probability regardless of any features to estimate the sensitivity of the shared location. Formally speaking,

Definition 4.9 (Sensitivity). The sensitivity of a given loc being shared by $u$ is defined as: sensitivity $($ loc $)=p_{\text {loc }}^{u}(\cdot)$.

\subsection{Final datasets}

The final datasets used in our study is shown in Table III including the basic statistics on the final privacy decisions.

\begin{tabular}{|c|c|}
\hline Dataset & Statistics \\
\hline Twitter Dataset & $\begin{array}{c}D_{\text {Twitter }}=\text { TSet }_{\text {req }} \cup \text { TSet }_{\text {rec }} \\
\text { (\#accepted: 4,874; \#rejected: } 7,914)\end{array}$ \\
\hline Google+ Dataset & $\begin{array}{c}D_{\text {Google+ }}=\text { GSet } \\
\text { (\#accepted }: 21,798 ; \text { \#rejected }: 114,400)\end{array}$ \\
\hline \multirow[b]{2}{*}{ Location Dataset } & $D_{\text {Family: }}$ \#shared: 8,$241 ; \#$ not shared: 2,554 \\
\hline & DFriend: \#shared: 8,$030 ;$ \#not shared: 2,845 \\
\hline
\end{tabular}

Table III: Statistics of datasets.

\section{PPM: PRIVACY PREDICTION MODEL}

Before building the privacy prediction model, we have analyzed and proved the above defined behavioral analogs work rather well on the all the datasets listed in Table ஹII] [Dong et al. 2015].

In this section we talk about how we build the PPM using the above presented behavioral analogs of the following psychological antecedents:

- (1) Trustworthiness of the requester/ information audience

- (2) Sharing tendency of the receiver/ information holder

- (3) Sensitivity of the requested/shared information 


\section{- (4) Appropriateness of the request}

Note that we also include:

\section{- (5) Other contextual factors}

The PPM is aimed to help OSN users to manage their privacy by predicting their disclosure behavior and recommending privacy settings in line with this behavior. Specifically, based on these behavioral analogs of the psychological antecedents, we build a binary classification model that learns the influence of these features on privacy decisions (i.e. sharing/disclosure decisions, such as accept vs. reject, or share vs. not share).

\subsection{Machine learning outcomes}

We build a decision making model for each of the five datasets described in Table III One problem with the five datasets is they are imbalanced, that is, the number of accepts/shares is much larger or smaller than the number of rejects/not-shares. We employ the common machine learning practice - undersampling, to balance the sets by randomly sampling items from the "large class" to match the size of the "small class". We use an adapted 10-fold cross validation approach (detailed implementation is described in the discussion in Section 5.2) to split the training and testing datasets. The final results are averaged over the classification results in all the folds. The commonly used $F 1$ and $A U C$ are employed as evaluation metrics. $F 1$ is the harmonic mean of precision and recall, and $A U C$ is a statistic that captures the precision of the model in terms of the tradeoff between false positives and false negatives. The higher of these values, the better of the performance. We use several of the binary classification algorithms provided by Weka [Hall et al. 2009] to build our models, including J48, Naïve Bayes, Support Vector Machine (SVM), etc. Among them, J48 produced the best results in terms of both $F 1$ and $A U C$. The corresponding results are shown in Table IV As seen, our privacy decision making prediction model has a good performance (cf. [Swets 1988]).

\begin{tabular}{|c|c|c|c|}
\hline \multirow{2}{*}{ Dataset } & \multirow{2}{*}{ \#tuples } & \multicolumn{2}{|c|}{ Measurements } \\
\cline { 3 - 4 } & & $\mathrm{F} 1$ & AUC \\
\hline \hline$D_{\text {Twitter }}$ & 9,748 & 0.796 & 0.85 \\
\hline$D_{\text {Google+ }}$ & 43,596 & 0.898 & 0.899 \\
\hline$D_{\text {Family }}$ & 5,378 & 0.845 & 0.879 \\
\hline$D_{\text {Friend }}$ & 5,822 & 0.810 & 0.844 \\
\hline$D_{\text {Colleague }}$ & 11,054 & 0.737 & 0.752 \\
\hline
\end{tabular}

Table IV: Results of privacy decision making prediction model.

We further verify the effectiveness of each factor by testing the privacy decision making model without the corresponding factor. Specifically, Table $\mathrm{V}$ compares the performance of the decision making models with all features $(A l l)$ against their performance after removing the features belonging to each of the factors using J48: (1) trustworthiness; (2) sharing tendency; (3) sensitivity; (4) appropriateness; (5) contextual factors $\sqrt{4}$.

Table $\mathrm{V}$ shows that removing some factors may reduce the prediction performance. In line with our feature ranking results in [Dong et al. 2015], this is mainly true for the trustworthiness of requester and the follow tendency of the receiver as well as the appropriateness of the request in the

\footnotetext{
${ }^{4}$ As contextual factors are not studied in the friend requests scenario, we have no results for removing such factors from $D_{\text {Twitter }}$ and $D_{\text {Google+ }}$. Similarly, although trustworthiness of the audience is an important factor in the location sharing study, our privacy decision making model is built for audience separately (as these measures are repeated per scenario). Thus, trustworthiness is not a feature in $D_{\text {Family }}, D_{\text {Friend }}$ and $D_{\text {Colleague. }}$. Finally, the features belonging to appropriateness are difficult to split off from contextual factors in the location study, so those results are combined.
} 


\begin{tabular}{|c|c|c|c|c|c|c|}
\hline \multirow{2}{*}{ Dataset } & \multicolumn{6}{|c|}{ F1 } \\
\cline { 2 - 7 } & \multirow{2}{*}{ All } & \multicolumn{5}{|c|}{ removed features } \\
\cline { 2 - 7 } & & $(1)$ & $(2)$ & $(3)$ & $(4)$ & $(5)$ \\
\hline$D_{\text {Twitter }}$ & 0.796 & 0.784 & $\mathbf{0 . 7 5 1}$ & 0.796 & $\mathbf{0 . 7 6 7}$ & - \\
\hline$D_{\text {Google+ }}$ & 0.898 & 0.889 & $\mathbf{0 . 8 8 7}$ & 0.898 & 0.889 & - \\
\hline$D_{\text {Family }}$ & 0.845 & - & 0.840 & $\mathbf{0 . 8 3 3}$ & $\mathbf{0 . 8 3 3}$ \\
\hline$D_{\text {Friend }}$ & 0.810 & - & $\mathbf{0 . 7 9 8}$ & 0.802 & 0.800 \\
\hline$D_{\text {Colleague }}$ & 0.737 & - & 0.730 & $\mathbf{0 . 7 2 6}$ & 0.727 \\
\hline \hline \multirow{3}{*}{ Dataset } & \multicolumn{7}{|c|}{ AUC } \\
\cline { 2 - 7 } & \multirow{2}{*}{ All } & \multicolumn{7}{|c|}{ removed features } \\
\cline { 2 - 7 } & & $(1)$ & $(2)$ & $(3)$ & $(4)$ & $(5)$ \\
\hline$D_{\text {Twitter }}$ & 0.850 & 0.835 & $\mathbf{0 . 8 1 2}$ & 0.850 & $\mathbf{0 . 8 3 2}$ & - \\
\hline$D_{\text {Google+ }}$ & 0.899 & 0.892 & $\mathbf{0 . 8 9 1}$ & 0.898 & $\mathbf{0 . 8 9 0}$ & - \\
\hline$D_{\text {Family }}$ & 0.879 & - & 0.875 & $\mathbf{0 . 8 6 7}$ & 0.870 \\
\hline$D_{\text {Friend }}$ & 0.844 & - & 0.840 & 0.839 & $\mathbf{0 . 8 3 5}$ \\
\hline$D_{\text {Colleague }}$ & 0.752 & - & 0.748 & $\mathbf{0 . 7 4 3}$ & 0.745 \\
\hline \hline
\end{tabular}

Table V: Performance of using different feature sets in decision making prediction model.

friend requests scenario of Twitter and Google+. These factors are more important than the sensitivity factors, probably because we simply use user profile to quantify it. In the location sharing scenario, the sharing tendency of the user, the sensitivity of the location as well as the contextual factors are all important predictors that reduce the $F 1$ and $A U C$ values when excluded. For different types of recipients, the dominate factors to privacy decisions vary as well. For instance, when the recipients/audiences are colleagues and family members, the sensitivity of the shared location is more important. However, the information holder's sharing tendency has a bigger influence on the privacy decisions when the recipients are their friends.

\subsection{Discussion}

5.2.1. Class imbalance problem. As we mentioned earlier, class imbalance is a common but serious problem when building classification models. To deal with imbalanced datasets, oversampling and undersampling are the two most commonly used techniques that use a bias to select more samples from one class than from another in order to balance the dataset. Specifically, oversampling is to repeatedly draw samples from the smaller class until the two classes have the same size. This technique may results in biased distribution due to certain over-repeated samples from the smaller class. While, undersampling is to randomly select the same number of instances from the bigger class as the number of instances in the smaller class. As some instances in the bigger class are not included in the final dataset, it may lose some information and result in a biased distribution. In our approach, we choose to use undersampling because of another approach adopted - adapted 10 fold cross validation. Traditional $k$-fold cross validation splits the same balanced dataset into $k$ parts, then each time uses 1 part of the dataset as testing dataset and the remaining $k-1$ parts as training dataset to evaluate the performance. This process repeats $k$ times with different part selected as testing dataset and the final results are averaged over the $k$ folds. In our work, instead of using the same balanced dataset and repeats 10 times, we built a different balanced dataset using undersampling each time and applied 10-fold cross validation. In this way, all the balanced datasets together may cover all or the majority of the instances in the bigger class. The results are actually averaged over the 100 different training-testing datasets, which can largely reduce the potential bias caused by undersampling.

5.2.2. Missing values in location datasets. There are a few differences when we built PPM for Google+/Twitter datasets and the three location datasets. In Google+ and Twitter datasets, each instance has the same number of attributes, represented by the measurements we defined for the be- 
havioral analogs of those psychological antecedents, including trustworthiness, sharing tendency, sensitivity and appropriateness. Thus, any machine learning algorithms can be directly applied on the datasets. For the three location datasets, however, the disclosure/sharing tendency will be measured by the overall sharing probability or the $\alpha$-conditional sharing probability, which is based on the contextual variables, e.g. companion, time, emotion. As described in Table II the five locationsharing scenarios we studied used different subsets of these contextual variables. By putting the data from these fives studies together, we thus create missing values for some of the attributes. We deal with this problem using the embedded ReplaceMissingValues filter in Weka. That is, Weka will deal with the missing values using different mechanisms given the different machine learning algorithms. Internally, $J 48$ replaces the missing values based on the weighted average value proportional to the frequencies of the non-missing values. As $J 48$ produces the best results not only on all the three location datasets, but also on Google+ and Twitter datasets, we may claim that the way missing values are solved by 48 is acceptable and may be a preferable approach compared with other possible approaches. Besides, the best performance of PPM achieved by $J 48$ does not purely rely on its mechanism of dealing with missing values.

5.2.3. PPM on simulation datasets. Table IV shows that PPM produces better results on the $D_{\text {Google+ }}$ dataset than on the $D_{\text {Twitter }}$ dataset in terms of both F1 score and AUC. As we mentioned earlier, the Google+ dataset simulates the "friend request" scenario more accurately than the Twitter dataset, as the chronological order of friendship invitations is captured in our Google+ dataset but not in the Twitter dataset. The better performance of PPM on the more accurate dataset arguably demonstrates the effectiveness of the proposed privacy prediction model.

\section{LIMITATIONS}

In our studies, we demonstrated that the factors identified in the PPM are essential in predicting users' disclosure/sharing behavior. The ultimate goal of the PPM is to give personalized privacy decision support to overwhelmed OSN users, and our first limitation is that we did not actually implement such a decision support system. We plan to do this in our future work, and point to [Knijnenburg 2015] for initial results in the context of recommender systems rather than OSNs. Aside from this, there are some other limitations with regard to our study that can be accounted for in future work.

The first limitation is our datasets: users' real sharing behavior data (rather than questionnaire data) is more accurate in identifying true factors that affect user decision making. We make use of a large dataset of Twitter and Google+ users to analyze such real behavior, but we use an imperfect proxy of users' actual "friend requests". Moreover, the location sharing data is based on imagined scenarios rather than real sharing behavior. This work takes a first step towards comprehensively and accurately study the factors affecting users' privacy decision making; in the future we hope to study these factors using real behavioral data such as true friend request acceptance and location sharing behaviors on social network sites.

The second limitation involves the identified factors. We analyzed the most important factors in privacy decision making as identified by existing work; but the study of factors determining privacy decisions has been far from comprehensive in the past. To create a more generally applicable model, we also specifically selected factors that are applicable to a wide range of privacy decision making scenarios in different social media, not restricted to online social networks. Consequently, we may miss system/domain-specific factors that could have a significant influence on privacy decision making. Finally, we tried to focus on very simple behavioral analogs in our study; more sophisticated analogs could likely be identified that further increase the prediction performance.

Our third limitation is that our privacy decision making prediction model is built as a binary classifier. In practical applications of, say, a "privacy recommender", it is not always appropriate to make "hard" recommendations to users, e.g., to pervasively recommend either "accept" or "reject". Instead, it might be more applicable to calculate a "privacy risk" score based on the identified factors 
and let user to make the final decision based on the score, or to only intervene when the calculated risk passes a certain (user-defined) threshold.

\section{DESIGN IMPLICATIONS AND CONCLUSION}

In this paper, we developed a generic Privacy Prediction Model based on known psychological antecedents of privacy decision making. We operationalized the PPM by identifying behavioral antecedents of the psychological factors, and analyzed how these factors influenced privacy decisions in several real-world and collected datasets. While our work does not move beyond prediction, it proves the feasibility of an automated tool to predict users' disclosure/sharing behavior. Such a tool can help ease the burden on the user, who on most OSNs has to make an almost unreasonable number of privacy decisions. Our investigation specifically led to the following important observations that may affect future design of OSNs:

- Consistent with previous studies in information sharing, who is the information audience is an important factor deciding if the information will be shared. Similarly, in the scenario of information requests, who is the requester determines the trust from the receiver, therefore determines the final privacy decision making outcome.

- As self-representation is one of the main purposes of OSNs, users' privacy decision making does not exclusively depend on their privacy concern, but more generally on the tradeoff between privacy and self-presentation. We have captured this in the definition of sharing tendency.

- Sensitivity is not an objective concept; it varies with audience. For example, in our location sharing preference study, locations such as Bar, Casino are more sensitive if shared to Colleague or Family compared to Friend. This effect may be captured by the appropriateness of the request.

- Although the assumption of "rationality" in privacy decision making has been criticized by many studies, users do consider the appropriateness of the request/sharing activity. Consistent with our intuition and previous studies, users tend to share information with or accept requests when this is appropriate in the current context.

- Contextual information is an indispensable factor in privacy decision making, primarily due to its effect on appropriateness. However, the effect of different contextual factors varies.

Concluding, privacy decision making is a trade-off between the potential benefit and risk; a tradeoff that is rather difficult for users to make. Our privacy decision making prediction model combines several important psychological and contextual factors that influence this tradeoff, and learns their functionality by building a binary classifier. The proposed privacy decision making prediction model produces good results based on the five identified factors, and can be used to assist users to protect their privacy in online social networks.

\section{REFERENCES}

A Acquisti and Ralph Gross. 2006. Imagined communities: Awareness, information sharing, and privacy on the Facebook. In In 6th Workshop on Privacy Enhancing Technologies. 36-58.

Alessandro Acquisti and Jens Grossklags. 2005. Privacy and rationality in individual decision making. IEEE Security \& Privacy 2 (2005), 24-30.

Alessandro Acquisti, Leslie K John, and George Loewenstein. 2012. The Impact of Relative Standards on the Propensity to Disclose. Journal of Marketing Research 49, 2 (2012), 160-174. DOI : http://dx.doi.org/10.1509/jmr.09.0215

Anne Adams. 2000. Multimedia Information Changes the Whole Privacy Ballgame. In Proceedings of the Tenth Conference on Computers, Freedom and Privacy: Challenging the Assumptions (CFP '00). ACM, 25-32.

Abdullah Al Hasib. 2009. Threats of online social networks. IJCSNS International Journal of Computer Science and Network Security 9, 11 (2009), 288-293.

Douglas Baker, Nicole Buoni, Michael Fee, and Caroline Vitale. 2011. Social networking and its effects on companies and their employees. Retrieved November 15 (2011).

R. Balebako, P. G. Leon, J. Mugan, A. Acquisti, L. F. Cranor, and N. Sadeh. 2011. Nudging users towards privacy on mobile devices. In CHI 2011 workshop on Persuasion, Influence, Nudge and Coercion Through Mobile Devices. Vancouver, Canada, 23-26. http://www.andrew.cmu.edu/user/jmugan/Publications/chiworkshop.pdf 
Gaurav Bansal, Fatemeh Zahedi, and David Gefen. 2008. The Moderating Influence of Privacy Concern on the Efficacy of Privacy Assurance Mechanisms for Building Trust: A Multiple-Context Investigation. In ICIS 2008 Proceedings. Paris, France. http://aisel.aisnet.org/icis2008/7

Solon Barocas and Helen Nissenbaum. 2009. On notice: The trouble with Notice and Consent. In Proceedings of the Engaging Data Forum: The First International Forum on the Application and Management of Personal Electronic Information. http://www.nyu.edu/pages/projects/nissenbaum/papers/ED_SII_On_Notice.pdf

Laura Becker and Key Pousttchi. 2012. Social Networks: The Role of Users' Privacy Concerns. In Proceedings of the 14th International Conference on Information Integration and Web-based Applications \& Services (IIWAS '12). ACM, 187195. DOI : http://dx.doi.org/10.1145/2428736.2428767

Michael Benisch, Patrick Gage Kelley, Norman Sadeh, and Lorrie Faith Cranor. 2011. Capturing location-privacy preferences: quantifying accuracy and user-burden tradeoffs. Personal Ubiquitous Computing 15, 7 (Oct. 2011), 679-694. DOI : http://dx.doi.org/10.1007/s00779-010-0346-0

Katrin Borcea-Pfitzmann, Andreas Pfitzmann, and Manuela Berg. 2011. Privacy $3.0:=$ Data Minimization + User Control + Contextual Integrity. Information Technology 53, 1 (2011), 34-40. DOI : http://dx.doi.org/10.1524/itit.2011.0622

Joanna Brenner and Aaron Smith. 2013. 72\% of Online Adults are Social Networking Site Users. PewResearch Internet Project (2013).

Ramón Compañó and Wainer Lusoli. 2010. The Policy Maker's Anguish: Regulating Personal Data Behavior Between Paradoxes and Dilemmas. In Economics of Information Security and Privacy, Tyler Moore, David Pym, and Christos Ioannidis (Eds.). Springer US, 169-185. 10.1007/978-1-4419-6967-5_9

Sunny Consolvo, Ian E. Smith, Tara Matthews, Anthony LaMarca, Jason Tabert, and Pauline Powledge. 2005. Location Disclosure to Social Relations: Why, when, \& What People Want to Share. In Proceedings of the SIGCHI Conference on Human Factors in Computing Systems (CHI '05). ACM, 81-90.

Mary J. Culnan. 1993. "How Did They Get My Name?": An Exploratory Investigation of Consumer Attitudes toward Secondary Information Use. MIS Quarterly 17, 3 (1993), 341-363. DOI :http://dx.doi.org/10.2307/249775

Joan DiMicco, David R. Millen, Werner Geyer, Casey Dugan, Beth Brownholtz, and Michael Muller. 2008. Motivations for Social Networking at Work. In Proceedings of the 2008 ACM Conference on Computer Supported Cooperative Work (CSCW '08). ACM, 711-720. DOI : http://dx.doi.org/10.1145/1460563.1460674

Cailing Dong, Hongxia Jin, and Bart P. Knijnenburg. 2015. Predicting Privacy Behavior on Online Social Networks. In Proceedings of the Ninth International Conference on Web and Social Media, ICWSM 2015, University of Oxford, Oxford, UK, May 26-29, 2015. 91-100. http://www.aaai.org/ocs/index.php/ICWSM/ICWSM15/paper/view/10554

Nicole Ellison, Charles Steinfield, and Cliff Lampe. 2007. The Benefits of Facebook "Friends: " Social Capital and College Students' Use of Online Social Network Sites. Journal of Computer-Mediated Communication (2007), 1143-1168.

Lujun Fang and Kristen LeFevre. 2010. Privacy Wizards for Social Networking Sites. In Proceedings of the 19th International Conference on World Wide Web (WWW'10). ACM, 351-360.

Neil Zhenqiang Gong, Wenchang Xu, Ling Huang, Prateek Mittal, Emil Stefanov, Vyas Sekar, and Dawn Song. 2011. Evolution of Social-Attribute Networks: Measurements, Modeling, and Implications using Google+. In Proceedings of the 2012 ACM Conference on Internet Measurement Conference (IMC'11). 131-144.

Ralph Gross and Alessandro Acquisti. 2005. Information Revelation and Privacy in Online Social Networks. In Proceedings of the 2005 ACM Workshop on Privacy in the Electronic Society (WPES '05). ACM, 71-80. DOI : http://dx.doi.org/10.1145/1102199.1102214

Mark Hall, Eibe Frank, Geoffrey Holmes, Bernhard Pfahringer, Peter Reutemann, and Ian H. Witten. 2009. The WEKA Data Mining Software: An Update. SIGKDD Explorations 11, 1 (2009).

L. Harris, A.F. Westin, and Associates. 1998. Personalized Marketing and Privacy on The Net: What Consumers Want. Privacy and American Business Newsletter. (1998).

Ai Ho, Abdou Maiga, and Esma Aïmeur. 2009. Privacy protection issues in social networking sites.. In AICCSA. IEEE, 271-278.

Chiung-wen Hsu. 2006. Privacy concerns, privacy practices and web site categories: Toward a situational paradigm. Online Information Review 30, 5 (2006), 569-586. DOI : http://dx.doi.org/10.1108/14684520610706433

Maritza Johnson, Serge Egelman, and Steven M. Bellovin. 2012. Facebook and privacy: it's complicated. In Proc. of the 8th Symposium on Usable Privacy and Security. ACM, Pittsburgh, PA. DOI : http://dx.doi.org/10.1145/2335356.2335369

Adam N. Joinson. 2008. Looking at, Looking Up or Keeping Up with People?: Motives and Use of Facebook. In Proceedings of the SIGCHI Conference on Human Factors in Computing Systems (CHI '08). ACM, 1027-1036. DOI :http://dx.doi.org/10.1145/1357054.1357213

Sanjay Kairam, Mike Brzozowski, David Huffaker, and Ed Chi. 2012. Talking in circles: Selective Sharing in Google+. In Proceedings of the SIGCHI Conference on Human Factors in Computing Systems. ACM Press, Austin, TX, 1065-1074. DOI : http://dx.doi.org/10.1145/2207676.2208552 
Bart P Knijnenburg. 2013. Simplifying Privacy Decisions: Towards Interactive and Adaptive Solutions. In Proceedings of the Recsys 2013 Workshop on Human Decision Making in Recommender Systems (Decisions@ RecSys'13). Hong Kong, China, 40-41.

Bart Piet Knijnenburg. 2015. A user-tailored approach to privacy decision support. Ph.D. University of California, Irvine, United States - California. http://search.proquest.com/docview/1725139739/abstract

Bart P Knijnenburg and Hongxia Jin. 2013. The Persuasive Effect of Privacy Recommendations. In Twelfth Annual Workshop on HCI Research in MIS. Milan, Italy. http://aisel.aisnet.org/sighci2013/16

Bart P. Knijnenburg and Alfred Kobsa. 2013. Making Decisions About Privacy: Information Disclosure in ContextAware Recommender Systems. ACM Trans. Interact. Intell. Syst. 3, 3, Article 20 (Oct. 2013), 23 pages. DOI : http://dx.doi.org/10.1145/2499670

B. P. Knijnenburg and A. Kobsa. 2014. Increasing Sharing Tendency Without Reducing Satisfaction: Finding the Best Privacy-Settings User Interface for Social Networks. In ICIS 2014 Proceedings. Auckland, New Zealand.

Bart P. Knijnenburg, Alfred Kobsa, and Hongxia Jin. 2013a. Dimensionality of Information Disclosure Behavior. International Journal of Human-Computer Studies 71, 12 (Dec. 2013), 1144-1162.

Bart P. Knijnenburg, Alfred Kobsa, and Hongxia Jin. 2013b. Preference-based Location Sharing: Are More Privacy Options Really Better?. In Proceedings of the SIGCHI Conference on Human Factors in Computing Systems (CHI '13). ACM, 2667-2676.

Brian David Eoff Kyumin Lee and James Caverlee. 2011. Seven Months with the Devils: A Long-Term Study of Content Polluters on Twitter. In International AAAI Conference on Weblogs and Social Media (ICWSM).

Cliff Lampe, Nicole B. Ellison, and Charles Steinfield. 2008. Changes in Use and Perception of Facebook. In Proceedings of the 2008 ACM Conference on Computer Supported Cooperative Work (CSCW '08). ACM, 721-730.

Cliff Lampe, Rebecca Gray, Andrew T. Fiore, and Nicole Ellison. 2014. Help is on the Way: Patterns of Responses to Resource Requests on Facebook. In Proceedings of the 17th ACM Conference on Computer Supported Cooperative Work \& Social Computing (CSCW '14). ACM, 3-15. DOI : http://dx.doi.org/10.1145/2531602.2531720

Robert S Laufer and Maxine Wolfe. 1977. Privacy as a Concept and a Social Issue: A Multidimensional Developmental Theory. Journal of Social Issues 33, 3 (1977), 22-42. DOI : http://dx.doi.org/10.1111/j.1540-4560.1977.tb01880.x

Scott Lederer, Jason I. Hong, Anind K. Dey, and James A. Landay. 2004. Personal privacy through understanding and action: five pitfalls for designers. Personal and Ubiquitous Computing 8, 6 (2004), 440-454. DOI : http://dx.doi.org/10.1007/s00779-004-0304-9

Scott Lederer, Jennifer Mankoff, and Anind K. Dey. 2003. Who Wants to Know What when? Privacy Preference Determinants in Ubiquitous Computing. In CHI '03 Extended Abstracts on Human Factors in Computing Systems (CHI EA '03). ACM, 724-725. DOI : http://dx.doi.org/10.1145/765891.765952

Kevin Lewis, Jason Kaufman, and Nicholas Christakis. 2008. The taste for privacy: An analysis of college student privacy settings in an online social network. Journal of Computer-Mediated Communication 14, 1 (2008), 79-100.

Yabing Liu, Krishna P. Gummadi, Balachander Krishnamurthy, and Alan Mislove. 2011. Analyzing facebook privacy settings: user expectations vs. reality. In In Proceedings of the 2011 ACM SIGCOMM conference on Internet measurement conference. ACM, 61-70.

Mary Madden. 2012. Privacy management on social media sites. Technical Report. Pew Internet \& American Life Project, Pew Research Center, Washington, DC. http://www.pewinternet.org/2012/02/24/privacy-management-on-social-media-sites/

M. Madejski, M. Johnson, and S.M. Bellovin. 2012. A study of privacy settings errors in an online social network. In 2012 IEEE International Conference on Pervasive Computing and Communications Workshops (PERCOM Workshops). Lugano, Switzerland, 340-345. DOI : http://dx.doi.org/10.1109/PerComW.2012.6197507

Helen Nissenbaum. 2009. Privacy in context: Technology, policy, and the integrity of social life. Stanford University Press, Stanford, CA.

Helen Nissenbaum. 2011. A Contextual Approach to Privacy Online. Daedalus 140, 4 (2011), $32-48$. DOI : http://dx.doi.org/10.1162/DAED_a_00113

Patricia A. Norberg, Daniel R. Horne, and David A. Horne. 2007. The privacy paradox: Personal information disclosure intentions versus behaviors. Journal of Consumer Affairs 41, 1 (2007), 100-126.

Judith S. Olson, Jonathan Grudin, and Eric Horvitz. 2005. A Study of Preferences for Sharing and Privacy. In CHI 'O5 Extended Abstracts on Human Factors in Computing Systems (CHI EA '05). ACM, 1985-1988.

Xinru Page, Alfred Kobsa, and Bart P. Knijnenburg. 2012. Don't Disturb My Circles! Boundary Preservation Is at the Center of Location-Sharing Concerns. In Proceedings of the Sixth International AAAI Conference on Weblogs and Social Media. Dublin, Ireland, 266-273. http://www.aaai.org/ocs/index.php/ICWSM/ICWSM12/paper/view/4679

Gautham Pallapa, Sajal K. Das, Mario Di Francesco, and Tuomas Aura. 2014. Adaptive and context-aware privacy preservation exploiting user interactions in smart environments. Pervasive and Mobile Computing 12 (June 2014), 232-243. DOI : http://dx.doi.org/10.1016/j.pmcj.2013.12.004 
Ramprasad Ravichandran, Michael Benisch, Patrick Kelley, and Norman Sadeh. 2009. Capturing Social Networking Privacy Preferences:. In Privacy Enhancing Technologies, Ian Goldberg and Mikhail Atallah (Eds.). Lecture Notes in Computer Science, Vol. 5672. Springer Berlin / Heidelberg, 1-18. 10.1007/978-3-642-03168-7_1

Consumer Reports. 2012. Facebook \& your privacy: Who sees the data you share on the biggest social network? (2012). http://www.consumerreports.org/cro/magazine/2012/06/facebook-your-privacy

Norman Sadeh, Jason Hong, Lorrie Cranor, Ian Fette, Patrick Kelley, Madhu Prabaker, and Jinghai Rao. 2009. Understanding and capturing people's privacy policies in a mobile social networking application. Personal and Ubiquitous Computing 13, 6 (2009), 401-412. DOI : http://dx.doi.org/10.1007/s00779-008-0214-3

Kim Bartel Sheehan. 2002. Toward a typology of Internet users and online privacy concerns. The Information Society 18,1 (2002).

N. Craig Smith, Daniel G. Goldstein, and Eric J. Johnson. 2013. Choice Without Awareness: Ethical and Policy Implications of Defaults. Journal of Public Policy \& Marketing 32, 2 (2013), 159-172. DOI : http://dx.doi.org/10.1509/jppm.10.114

Daniel J. Solove. 2013. Privacy Self-Management and the Consent Dilemma. Harvard Law Review 126 (2013), $1880-1903$. http://papers.ssrn.com/abstract=2171018

J. A. Swets. 1988. Measuring the accuracy of diagnostic systems. Science (New York, N.Y.) 240, 4857 (June 1988), 12851293.

Karen Tang, Jason Hong, and Dan Siewiorek. 2012. The Implications of Offering More Disclosure Choices for Social Location Sharing. In Proceedings of the SIGCHI Conference on Human Factors in Computing Systems (CHI '12). ACM, 391-394.

Karen Tang, Jialiu Lin, Jason Hong, Daniel Siewiorek, and Norman Sadeh. 2010. Rethinking location sharing: exploring the implications of social-driven vs. purpose-driven location sharing. In Proc. UbiComp 2010. Copenhagen, Denmark, 85-04. DOI : http://dx.doi.org/10.1145/1864349.1864363

Humphrey Taylor. 2003. Most people are "privacy pragmatists" who, while concerned about privacy, will sometimes trade it off for other benefits. The Harris Poll 17, 19 (2003).

Eran Toch, Justin Cranshaw, Paul Hankes Drielsma, Janice Y. Tsai, Patrick Gage Kelley, James Springfield, Lorrie Cranor, Jason Hong, and Norman Sadeh. 2010. Empirical models of privacy in location sharing. In Proc. of the 12th ACM intl. conference on Ubiquitous computing. ACM Press, Copenhagen, Denmark, 129-138. DOI : http://dx.doi.org/10.1145/1864349.1864364

Jason Watson, Andrew Besmer, and Heather Richter Lipford. 2012. +Your circles: sharing behavior on Google+. In Proc. of the 8th Symposium on Usable Privacy and Security. ACM, Pittsburgh, PA. DOI : http://dx.doi.org/10.1145/2335356.2335373

Allison Woodruff, Vasyl Pihur, Sunny Consolvo, Lauren Schmidt, Laura Brandimarte, and Alessandro Acquisti. 2014. Would a privacy fundamentalist sell their DNA for $\$ 1000 \ldots$ if nothing bad happened as a result? The Westin categories, behavioral intentions, and consequences. In Symposium on Usable Privacy and Security (SOUPS). https://www.usenix.org/system/files/conference/soups2014/soups14-paper-woodruff.pdf

H. Xu, T. Dinev, H. J Smith, and P. Hart. 2008. Examining the formation of individual's privacy concerns: Toward an integrative view. In ICIS 2008 Proceedings. Paris, France.

Kathryn Zickuhr. 2012. Three-quarters of smartphone owners use location-based services. Technical Report. Pew Research Center. http://pewinternet.org/ /media//Files/Reports/2012/PIP_Location_based_services_2012_Report.pdf 